\title{
In situ HR-TEM and Simulation of Si Field Emitter Tips under Field Evaporation
}

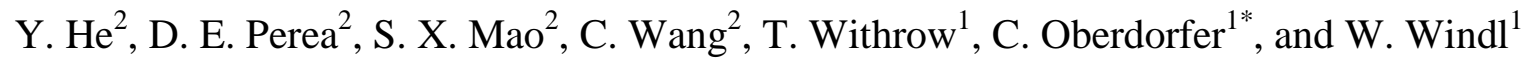 \\ 1. Department of Materials Science and Engineering, The Ohio State University, Columbus, USA \\ 2. Environmental Molecular Sciences Laboratory, Pacific Northwest National Laboratory, Richland, \\ USA \\ * Corresponding author: oberdorfer.4@ osu.edu
}

Understanding of atomistic processes involved with the field evaporation of atoms from field emitter tips is crucial to achieve atomic precision in microscopic manipulation and atom probe tomography (APT). The applied electric field in APT is in the order of $\sim 10^{10} \mathrm{~V} / \mathrm{m}$ which is sufficient to induce the ionization of surface atoms followed by ionic emission from the surface. However, what is going on at the nano to atomic scale, first, when an electric field applies, and second, when the field increases to facilitate field evaporation, is still elusive.

By virtue of a bias voltage applied to an atomically sharp needle in a transmission electron microscope (TEM) using a biasing specimen holder, we were able to generate a sufficiently strong electric field to induce field evaporation of $\mathrm{Si}$ and directly capture the resultant changes in local surface bonding in situ at atomic resolution (Fig. 1). It is found that surface bonding characteristics govern the evaporation sequence of surface atoms which manifest in a remarkable orientation effect with the evaporation proceeding normal to the (001) plane and parallel to the (111) plane. A careful detailed inspection of the obtained images shows that the outmost $\mathrm{Si}$ atoms are distorted upon bias with respect to the original tetrahedral configuration. The Si surface atom bonds are observed to stretch away from the substrate upon field excitation, forming clusters before evaporation (Fig. 2).

In order to investigate the observed surface reconstruction effect and the striking evaporation order along the (111) lattice planes in more detail, we conducted atomistic simulations of the Si (100) field evaporation with TAPSim-MD [1]. TAPSim-MD combines the classic electrostatic APT modeling approach on the basis of finite elements (FE) [2] with molecular dynamic (MD) calculations in LAMMPS [3]. Considering the Tersoff potential from Mishin [4] for the calculation of the Si bonds, we calculate the same characteristic surface reconstruction as is observed in the experiment. However, this is only possible if the MD simulation is conducted at an elevated temperature. In contrast, by including field-induced forces in the MD calculation, severe strain is added to the whole emitter structure with the surface atoms at the emitter apex experiencing this in particular. We find this field-induced local strain can then facilitate surface reconstruction without having to include an elevated temperature.

In another aspect the covalent nature of the Si bonds suggests that the actual evaporation field of Si has a pronounced dependence on local bonding environment. Therefore, we investigated the Si evaporation sequence considering site-dependent cohesive energies derived from MD and compared results with the application of a globally fixed average evaporation field.

In summary, simulations support the experimental findings, and both together are providing fundamental insight in the complex nature of field evaporation and ultimately provide strategies for better control over field evaporation for optimized applications as well as addressing data reconstruction aberrations. 


\section{References:}

[1] C Oberdorfer et al., Mater. Charact. 146 (2018), p. 324.

[2] F Vurpillot and C Oberdorfer, Ultramicroscopy 159 (2015), p. 202.

[3] S Plimpton, J. Comput. Phys. 117 (1995), p. 19.

[4] G.P.P. Pun and Y. Mishin, Phys. Rev. B 95 (2017), 224102.

[5] L. Yao, T. Withrow, O.D. Restrepo, W. Windl, and E.A. Marquis, Appl. Phys. Lett. 107 (2015), 241602.

[6] The authors acknowledge financial support from the Air Force Office of Scientific Research, Award Number FA9550-14-1-0249 and computational support by the Ohio Supercomputer Center, Grant Number PAS0072. CO gratefully acknowledges support from the Alexander von Humboldt Foundation through the Feodor-Lynen research fellowship. A portion of the research was performed using EMSL (grid.436923.9), a DOE Office of Science User Facility sponsored by the Office of Biological and Environmental Research.

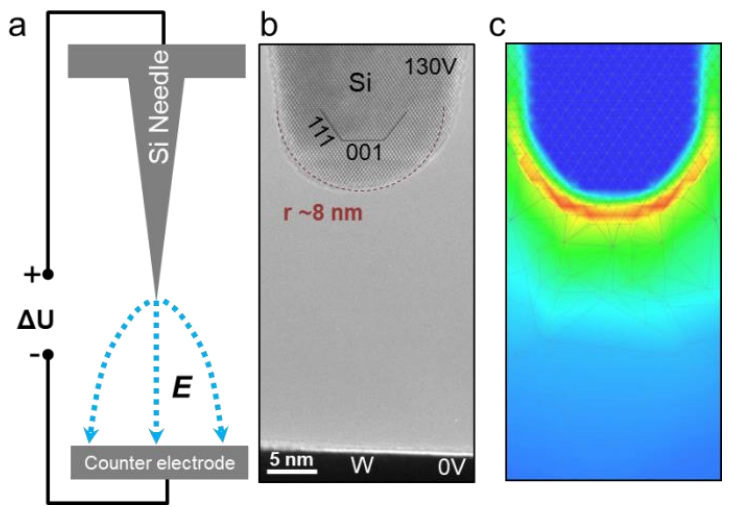

Figure 1. Experimental setup for the in situ TEM field evaporation measurement: (a) A voltage bias is applied to a Si field emitter sample while it is inserted in the TEM. (b) In this way, the field induced evaporation of atoms from the sample surface can be observed in situ at atomic resolution. (c) The numeric solution for the electric field in the vicinity of the emitter surface shows that the region with decisively elevated field strength is practically confined to the apex.
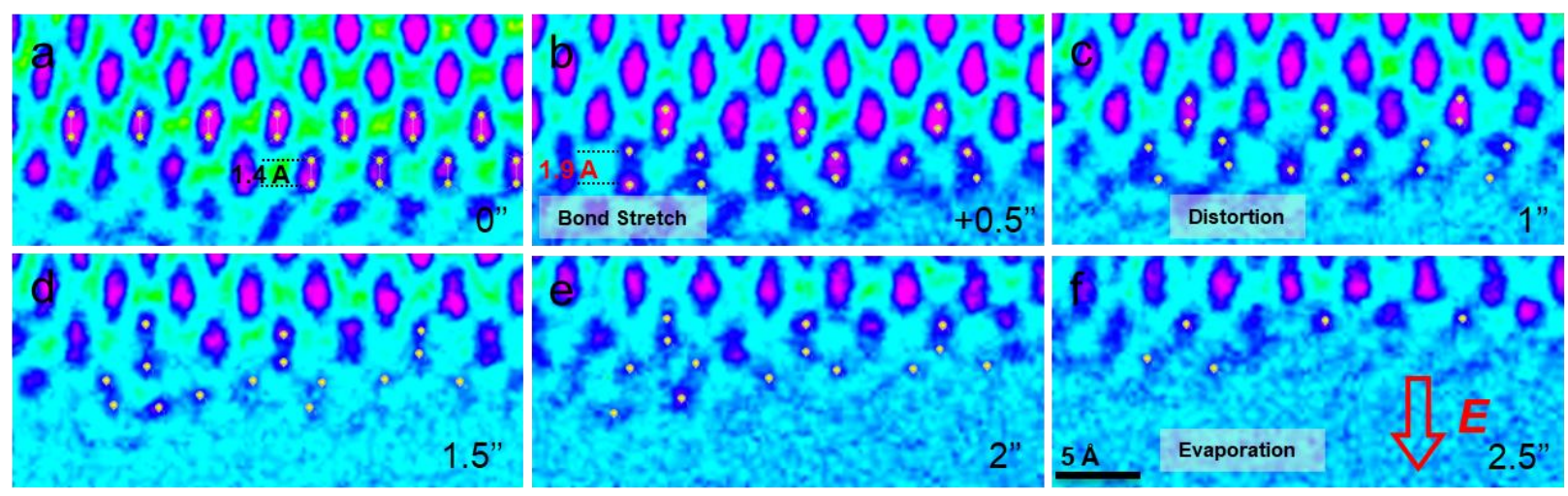

Figure 2. Sequential images of Si-Si dumbbell rupture process of [001] tip field evaporation. Surface bonds (a) are highly stretched (>30\% for the dumbbell) upon field application (b). The "surface thickness" or "penetration depth" of the electric field is about 3-4 atom layers or the outmost tetrahedral. When stretching away from the substrate, the energized surface atoms tend to bond locally with each other before evaporation (c, d). Subsequently, the evaporation proceeds normally to the (001) plane by rupture of dumbbells. 